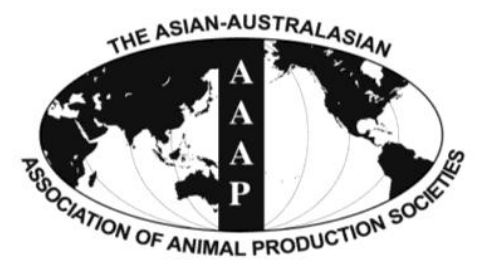

Asian-Aust. J. Anim. Sci.

Vol. 25, No. 4 : 592 - 596

April 2012

www.ajas.info

http://dx.doi.org/10.5713/ajas.2011.11204

\title{
Simple Spot Method of Image Analysis for Evaluation of Highly Marbled Beef
}

\author{
M. Irie* and K. Kohira ${ }^{1}$ \\ Faculty of Agriculture, University of Miyazaki, Miyazaki 889-1601, Japan
}

\begin{abstract}
The simple method of evaluating highly marbled beef was examined by image analysis. The images of the cross section at the 6 to 7 th rib were obtained from 82 carcasses of Wagyu cattle. By using an overall trace method, the surrounding edges of the longissimus thoracis and three muscles were traced automatically and manually with image analysis. In a spot method, 3 to 5 locations ( 2.5 or $3.0 \mathrm{~cm}$ in diameter) for each muscle were rapidly selected with no manual trace. The images were flattened, binarized, and the ratio of fat area to muscle area was determined. The correlation coefficients for marbling between different muscles, and between the overall trace and the spot methods were 0.55 to 0.81 between different muscles and 0.89 to 0.97 , respectively. These results suggested that the simple spot method is speedy and almost as useful as the overall trace method as a measuring technique for beef marbling in loin muscles, especially for highly marbled beef. (Key Words : Image Analysis, Meat, Marbling, Wagyu)
\end{abstract}

\section{INTRODUCTION}

The image analysis method has been developed for objective evaluation of meat quality (Tan, 2004). It can also be applied for the evaluation of meat marbling (Albrecht et al., 1996; Gerrard et al., 1996; Kuchida et al., 2000; Yang et al., 2006). Marbling in beef is an important factor that influences taste, juiciness, tenderness and flavor (Okitani, 2002; Platter et al., 2005). Wagyu beef, in particular, served in the Japanese restaurants in Asia, China, Europe and USA often possesses abundant marbling, the market rate thus sometimes exceeds $\$ 20 / \mathrm{kg}$ of carcass or $\$ 100 / \mathrm{kg}$ of retail loin, depending mainly on marbling. Although graders are professionally trained, subjective evaluation often lead to inconsistencies and variations (Cross et al., 1983).

The image analysis method for marbling has been developed but not yet been applied in meat grading for highly marbled beef in Asia, because it is labor consuming for capture of image and manual operation of correction and needs a special apparatus. Original image analysis method is extremely rapid, and its automation has been advanced. Image analysis for beef marbling is used in North America

\footnotetext{
* Corresponding Author : M. Irie. Tel : +81-985-58-7202, Fax : +81-985-7202, E-mail : irie@cc.miyazaki-u.ac.jp

1 National Livestock Breeding Center, Fukushima 961-8511, Japan.

Submitted May 16, 2011; Accepted Sept. 20, 2011; Revised Nov. 3, 2011
}

(personal comm). However, when the muscle outline is auto-traced for evaluation of high marbling, troublesome manual operation is often needed for distinguish between meat and fat, especially for highly marbled meat such as that of Wagyu beef.

Therefore, we designed a spot method that measures a small area of a constant part without the need for manual correction. Moreover, this method has the merit of evaluating the degree of marbling even when only partial images are obtained from a narrowly cut section to prevent abnormal diffusion of prions or when the evaluation of other portions such as the hip is required for determining the accurate commercial value. Further, we attempted to compare the overall trace method with the spot method in evaluating the marbling of each beef muscle.

\section{MATERIALS AND METHODS}

\section{Sample preparation}

Samples were obtained from carcasses of Wagyu (Japanese Black) cattle. Its beef is characterized by a unique ability to deposit large amounts of intramuscular fat (Zembayashi et al., 1988; Cameron et al., 1994). The materials included 82 cross sections at the 6 to 7 th rib of carcasses, which is the Japanese standard location for measuring marbling.

Photographs of the loin cuts including longissimus 


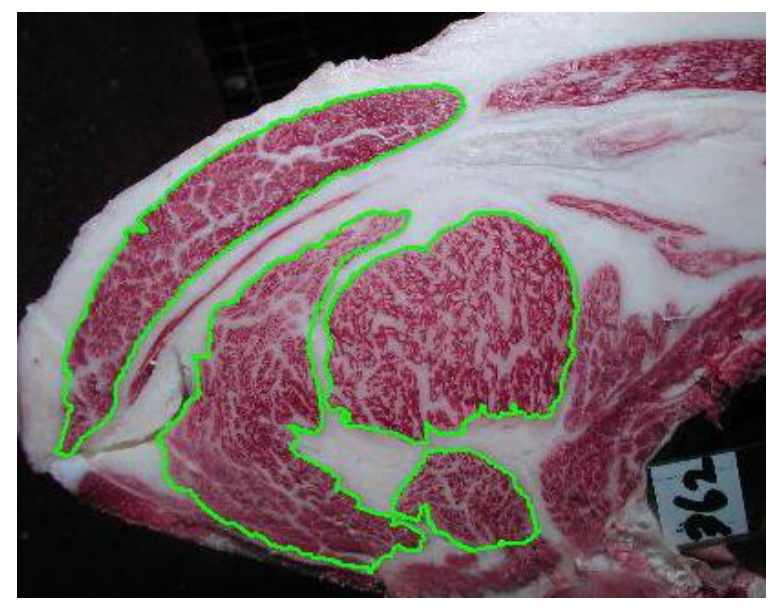

Figure 1. Overall trace method by image analysis. Muscle areas were surrounded by the boundary line which was drawn with automatic and manual traces by image analysis software.

thoracis, trapezius, semispinalis dorsi and semispinalis capitis muscles were taken with a scale using a digital camera (C-3100, Olympus Co., Tokyo, Japan) on the carcasses at meat markets. The flash unit in the digital camera was not used because the light reflectance of moisture on meat surface lead to error.

\section{Image analysis}

The photographic images were imported to a personal computer loaded with the image analysis software (Image Pro PLUS Ver. 4.0, Media Cybernetics, Inc., MD, USA). The images were calibrated with the scale in the photograph according to the software requirements. To eliminate subjective operator-to-operator differences, the measurements were performed by only 1 experienced operator.

In the overall trace method, the surrounding edges of the longissimus thoracis, trapezius, semispinalis dorsi and semispinalis capitis muscles were automatically traced by auto trace operation in the image analysis software (Figure 1). However, whenever error in the trace was detected on the monitor, manual trace was added. The images were edited in order to flatten the background and remove irregularities in the illumination by using the image analysis software. The suitable conditions of image analysis were well-studied prior to the present study (flatten filter $=100$ ). Then the images were binarized for fat and muscle, and ratio of fat area to muscle area was determined as the marbling index.

In the spot method, 3 to 5 locations $(3.0 \mathrm{~cm}$ in diameter) for longissimus thoracis muscle, 3 locations $2.5 \mathrm{~cm}$ in diameter for trapezius muscle, 2 locations $3.0 \mathrm{~cm}$ in diameter for semispinalis dorsi muscle and 1 location 2.5 $\mathrm{cm}$ in diameter for semispinalis capitis muscle were selected rapidly on the monitor (Figure 2). The images were

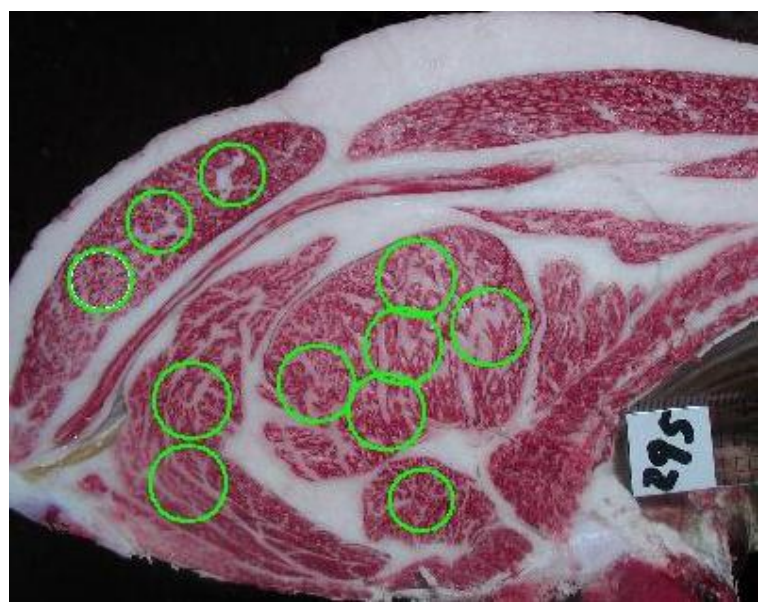

Figure 2. Spot method by image analysis. The areas were selected speedy in 3 and 2.5 centimeters in diameter for longissimus thoracis, trapezius, semispinalis dorsi and semispinalis capitis muscles.

flattened, binarized and measured for ratio of fat area to muscle area by the software. In the spot method, there was no additional manual trace.

\section{Statistical analysis}

Descriptive statistics and simple correlation coefficients were calculated using SPSS (SPSS 15.0J. Japan Inc, Tokyo, Japan).

\section{RESULTS AND DISCUSSION}

\section{Measurements by image analysis}

Table 1 shows the average, standard deviation, maximum and minimum values by image analysis. The measurements at the same muscles were similar between the methods. The slight differences in values by methods at the same muscle may be caused by slight differences in automatic treatment of image analysis and/or in marbling distribution between the surrounding and the center parts in muscle. But, this will not actually become a problem as shown the following.

\section{Correlation of the area and marbling between each muscle}

Table 2 shows the correlation coefficients between muscle areas. All correlation coefficients between muscle areas were above 0.5 . The areas of the longissimus thoracis muscle in particular were relatively strongly correlated with semispinalis dorsi muscle $(r=0.81)$, semispinalis capitis muscle $(r=0.72)$ and trapezius muscle $(r=0.63)(p>0.01)$.

These results indicate that the measurement of parameters of 1 muscle area, especially the longissimus thoracis muscle, can indicate the values for the other muscle areas. As the muscle area relates with the volume of meat, 
Table 1. Measurements of beef marbling (fat area ratio) between longissimus thoracis, trapezius, semispinalis dorsi and semispinalis capitis muscles by image analysis

\begin{tabular}{lllllc}
\hline & & Means & SD $^{1}$ & Minimum & Maximum \\
\hline Longissimus thoracis & Overall & 0.35 & 0.08 & 0.16 & 0.51 \\
& 5 spot & 0.37 & 0.09 & 0.17 & 0.55 \\
& 4 spot & 0.37 & 0.09 & 0.16 & 0.55 \\
Trapezius & 3 spot & 0.38 & 0.10 & 0.17 & 0.56 \\
\multirow{3}{*}{ Semispinalis dorsi } & Overall & 0.28 & 0.07 & 0.12 & 0.44 \\
\multirow{2}{*}{ Semispinalis capitis } & O spot & 0.28 & 0.08 & 0.10 & 0.52 \\
& Overall & 0.35 & 0.06 & 0.23 & 0.50 \\
& Overall & 0.35 & 0.07 & 0.21 & 0.50 \\
\hline
\end{tabular}

$\mathrm{n}=82 .{ }^{1}$ Standard deviations.

Table 2. Simple correlation coefficients of beef muscle area between longissimus thoracis, trapezius, semispinalis dorsi and semispinalis capitis muscles by image analysis

\begin{tabular}{lccc}
\hline & $\begin{array}{c}\text { Longissimus } \\
\text { thoracis }\end{array}$ & Trapezius & $\begin{array}{c}\text { Semispinalis } \\
\text { dorsi }\end{array}$ \\
\hline Trapezius & $0.63 * *$ & & \\
Semispinalis dorsi & $0.81^{* *}$ & $0.58^{* *}$ & \\
Semispinalis capitis & $0.72 * *$ & $0.55^{* *}$ & $0.74 * *$ \\
\hline $\mathrm{n}=82 . * * \mathrm{p}<0.001$. & & &
\end{tabular}

the formulas for determining beef yield grade in USA (AMSA) and Japan (JMGA) incorporate the area of the longissimus thoracis muscle.

Image analysis can measure longissimus muscle area and predict yield grade (Karnuah et al., 2001; Steiner et al., 2003). Determination of longissimus muscle area is fast and can be automate for lean beef (Steiner et al., 2003) but requires manual operation for highly marbled beef (Nade et al., 2004). Nade et al. (2004) reported that a new equation incorporating total muscle areas in the loin by image analysis is more suitable for Japanese beef yield grade than the present equation containing the longissimus muscle area only. A rough estimation of all muscle areas in the loin is quicker than an accurate measurement of each muscle area manually for yield grade of marbled beef.

Table 3 displays the correlation coefficients of marbling between muscles. In the overall trace method for marbling, the marbling in the longissimus thoracis muscle was relatively strongly correlated with semispinalis dorsi muscle $(\mathrm{r}=0.73)$, semispinalis capitis muscle $(\mathrm{r}=0.69)$ and trapezius muscle $(r=0.70)(p>0.01)$. Marbling in the loin was similar high correlation coefficient to muscle area. Although the marbling of 1 muscle was comparatively highly related with marbling of the other muscles in loin, the relationships may not always apply for other portions such as the hip (unpublished data). Meat packers in Japan gaze the marbling in other portions such as the hip, in addition to marbling in the loin.

Table 3. Simple correlation coefficients of beef marbling (fat area ratio) between longissimus thoracis, trapezius, semispinalis dorsi and semispinalis capitis muscles by image analysis

\begin{tabular}{|c|c|c|c|c|c|c|c|c|c|c|}
\hline & & \multicolumn{4}{|c|}{ Longissimus thoracis muscle } & \multicolumn{2}{|c|}{ Trapezius } & \multicolumn{2}{|c|}{ Semispinalis dorsi } & \multirow{2}{*}{$\begin{array}{c}\begin{array}{c}\text { Semispinalis } \\
\text { capitis }\end{array} \\
\text { Overall }\end{array}$} \\
\hline & & Overall $^{1}$ & $5 \mathrm{spot}^{2}$ & 4 spot & 3 spot & Overall & 3 spot & Overall & 2 spot & \\
\hline \multirow[t]{3}{*}{ Longissimus thoracis } & 5 spot & $0.97 * *$ & & & & & & & & \\
\hline & 4 spot & $0.97 * *$ & $0.99 * *$ & & & & & & & \\
\hline & 3 spot & $0.96^{* *}$ & $0.99 * *$ & $0.99 * *$ & & & & & & \\
\hline \multirow[t]{2}{*}{ Trapezius } & Overall & $0.70^{* *}$ & $0.68 * *$ & $0.69 * *$ & $0.67 * *$ & & & & & \\
\hline & 3 spot & $0.74 * *$ & $0.74 * *$ & $0.74 * *$ & $0.73 * *$ & $0.89 * *$ & & & & \\
\hline \multirow[t]{2}{*}{ Semispinalis dorsi } & Overall & $0.73^{* *}$ & $0.75 * *$ & $0.76^{* *}$ & $0.74 * *$ & $0.68 * *$ & $0.66 * *$ & & & \\
\hline & 2 spot & $0.68 * *$ & $0.69 * *$ & $0.70^{* * *}$ & $0.70 * *$ & $0.65 * *$ & $0.64 * *$ & 0.93 & & \\
\hline \multirow[t]{2}{*}{ Semispinalis capitis } & Overall & $0.69 * *$ & $0.72 * *$ & $0.73 * *$ & $0.72 * *$ & $0.53 * *$ & $0.58 * *$ & $0.74 * *$ & $0.79 * *$ & \\
\hline & 1 spot & $0.65^{* *}$ & 0.69 & $0.70 * *$ & $0.69^{* *}$ & $0.55^{* *}$ & $0.57 * *$ & $0.72 * *$ & $0.78 * *$ & $0.93 * *$ \\
\hline
\end{tabular}

$\mathrm{n}=82 . * *$ Correlation is significant at $\mathrm{p}<0.001 .{ }^{1}$ Overall trace method by image analysis. ${ }^{2}$ Spot method by image analysis. 


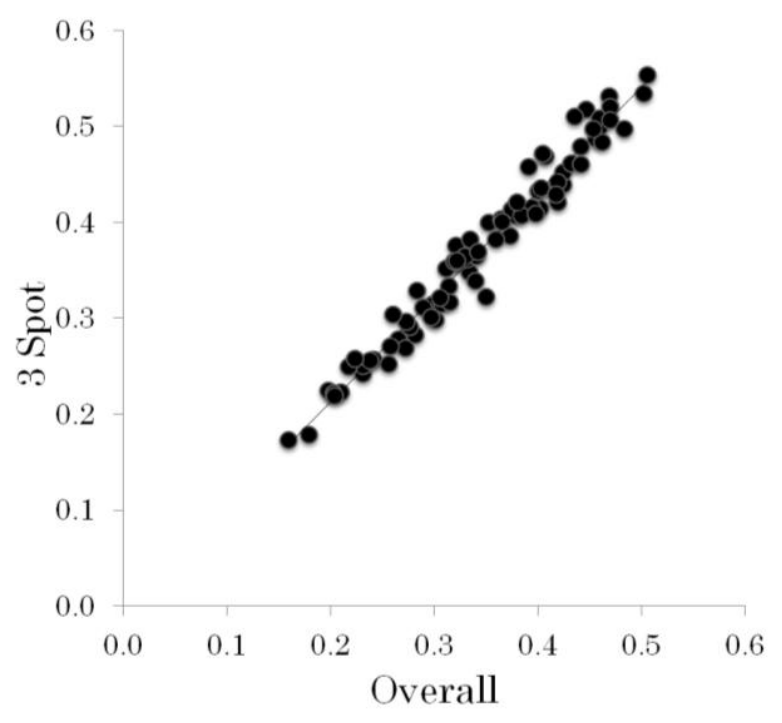

Figure 3. Relationship between measurements by overall trace and 3 spot methods for longissimus thoracis.

It was reported that fat area determined by image analysis related with lipid content $(\mathrm{r}=0.71$, Yang et al., 2006; $r=0.98$, Kuchida et al., 2000). Measurements of marbling by image analysis at meat markets can provide information on lipid contents and calories to the consumer.

\section{Relationships between the simple spot and overall trace methods}

Table 2 also shows that the relationships between the overall trace and spot methods. The spot method for beef marbling related strongly with the overall trace method (from minimum value $r=0.89$ to maximum value $r=0.97$ ) in all muscles. These results showed that the spot method can substitute the overall trace method. As an example, the relationship between the measurements by overall trace and 3 spot methods for longissimus thoracis is shown in Figure 3. These data are to be comparatively fitted by a straight line. Although the spot method cannot determine muscle area, it has some merits. The spot method does not entail the time-consuming manual operation of the overall method. Even when the overall image is not obtained because of a narrow cut, the spot method can make use of even a partial image. Because the constant area is measured, the spot method is used to measure only the fat area, thus measuring of 2 or more spots do not take time. This enables the miniaturization of the photographic device with constant illumination by a spot light, such as fiber-optic devices (Irie and Swatland, 1993). In addition, this method can be applied to other rounded parts such as the hip. Hip is second important parts for marbling evaluation. Some beef carcasses with high marbling at loin have occasionally low marbling at hip. When this method is combined with the CT image (Holló et al., 2007), the evaluation of the marbling on various tissues might speed up. Further study including new capture devices for spot method is needed for application to meat market.

\section{REFERENCES}

Albrecht, E., J. Wegner and K. Ender. 1996. A new technique for objective evaluation of marbling in beef. Fleischwirtschaft 76:1145-1148.

AMSA. 2001. Meat evaluation handbook. American Meat Science Association, Illinois, USA.

Cameron, P. J., M. Zembayashi, D. K. Lunt, T. Mitsuhashi, M. Mitsumoto, S. Ozawa and S. B. Smith. 1994. Relationship between Japanese beef marbling standard and intramuscular lipid in the M. longissimus thoracis of Japanese Black and American Wagyu cattle. Meat Sci. 38:361-364.

Cross, H. R., D. A. Gilliland, P. R. Durland and S. Seideman. 1983. Beef carcass evaluation by use of a video image analysis system. J. Anim. Sci. 57:910-917.

Gerrard, D. E., X. Gao and J. Tan. 1996. Determining beef marbling and color scores by image processing. J. Food Sci. 61:145-148.

Holló, G., E. Szűcs, J. Tőzsér, I. Holló and I. Repa. 2007. Application of X-ray computer tomography (CT) in cattle production. Asian-Aust. J. Anim. Sci. 20:1901-1908.

Irie, M. and H. J. Swatland. 1993. - Review - Fiber-optic methods and their applications to meat. Nippon Shokuhin Kogyou Gakkaishi 40:747-754.

JMGA. 1988. New beef carcass grading standards. Japan Meat Grading Association, Tokyo.

Karnuah, A. B., K. Moriya, N. Nakanishi, T. Nade, T. Mitsuhashi and Y. Sasaki. 2001. Computer image analysis for prediction of carcass composition from cross-sections of Japanese Black steers. J. Anim. Sci. 79:2851-2856.

Kuchida, K., S. Kono, K. Konishi, L. D. Van Vleck, M. Suzuki and S. Miyoshi. 2000. Prediction of crude fat content of longissimus muscle of beef using the ratio of fat area calculated from computer image analysis: comparison of regression equations for prediction using different input devices at different stations. J. Anim. Sci. 78:799-803.

Nade, T., A. B. Karnuah, Y. Masuda, S. Hirabara and K. Fujita. 2001. Estimation of carcass composition from the crosssection at rib-loin of Japanese Black steers by computer image analysis. Anim. Sci. J. 72:313-320.

Okitani, A. 2002. Factors determining the palatability of meat. Jpn. J. Nutr. Diet. 60:119-129.

Steiner, R., A. M. Wyle, D. J. Vote, K. E. Belk, J. A. Scanga, J. W. Wise, J. D. Tatum and G. C. Smith. 2003. Real-time augmentation of USDA yield grade application to beef carcasses using video image analysis. J. Anim. Sci. 81:22392246.

Tan, J. 2004. Meat quality evaluation by computer vision. J. Food Eng. 61:27-35.

Platter, W. J., J. D. Tatum, K. E. Belk, S. R. Koontz, P. L. Chapman and G. C. Smith. 2005. Effects of marbling and shear force on consumers' willingness to pay for beef strip loin steaks. J. Anim. Sci. 83:890-899. 
Yang, X. J., E. Albrecht, K. Ender, R. Q. Zhao and J. Wegner. 2006. Computer image analysis of intramuscular adipocytes and marbling in the longissimus muscle of cattle. J. Anim. Sci. 84:3251-3258.
Zembayashi, M., H. Nabeta and T. Mototsuji. 1988. Effects of breeds and nutritional planes on intramuscular lipid deposition of fattening of steers. Jpn. J. Zootech. Sci. 59:39-48. 